PROCEEDINGS OF THE

AMERICAN MATHEMATICAL SOCIETY

Volume 139, Number 2, February 2011, Pages 499-510

S 0002-9939(2010)10481-1

Article electronically published on July 15, 2010

\title{
CLASSIFICATION OF ORDER PRESERVING ISOMORPHISMS BETWEEN ALGEBRAS OF SEMICLASSICAL OPERATORS
}

\author{
HANS CHRISTIANSON
}

(Communicated by Matthew J. Gursky)

\begin{abstract}
Following the work of Duistermaat and Singer on isomorphisms of algebras of global pseudodifferential operators, we classify order preserving isomorphisms of algebras of microlocally defined semiclassical pseudodifferential operators. Specifically, we show that any such isomorphism is given by conjugation by $A=B F$, where $B$ is a microlocally elliptic semiclassical pseudodifferential operator and $F$ is a microlocally unitary $h$-FIO associated to the graph of a local symplectic transformation.
\end{abstract}

\section{INTRODUCTION}

In the study of pseudodifferential operators on manifolds, there are two important regimes to keep in mind. The first is a global study of pseudodifferential operators defined using the local Fourier transform on the cotangent bundle. If $X$ is a compact smooth manifold and $T^{*} X$ is the cotangent bundle with the local coordinates $\rho=(x, \xi)$, we study pseudodifferential operators with principal symbol homomgeneous at infinity in the $\xi$ variables. Let $Y$ be another compact smooth manifold of the same dimension as $X$, suppose there is an algebra isomorphism from the algebra of all pseudodifferential operators on $X$ (filtered by order) to the same algebra on $Y$, and suppose that isomorphism preserves the order of the operator. Then Duistermaat and Singer DuSi] have shown that this isomorphism is necessarily given by conjugation by an elliptic Fourier Integral Operator (FIO).

The other setting is the semiclassical or "small- $h$ " regime. One can study globally defined semiclassical pseudodifferential operators, but many times it is meaningful to study operators which are microlocally defined in some small set (see 92 for definitions). Then we think of the $h$ parameter as being comparable to $|\xi|^{-1}$ in the global, non-semiclassical regime. Thus the study of small- $h$ asymptotics in the microlocally defined regime should correspond to the study of high-frequency asymptotics in the global regime. We therefore expect a similar result to that presented in DuSi], although the techniques used in the proof will vary slightly.

Let $X$ be a smooth manifold, $\operatorname{dim} X=n \geq 2$, and assume $U \subset T^{*} X$ is a neighbourhood of $\rho_{0} \in T^{*} X$ so that $H^{1}(U, \mathbb{C})=0$. Let $Y$ be another smooth manifold, $\operatorname{dim} Y=n$. Let $V \subset T^{*} Y$ be a non-empty precompact open subset of the cotangent bundle of $Y$. Let $\Psi^{0} / \Psi^{-\infty}(U)$ denote the algebra of semiclassical

Received by the editors March 6, 2008 and, in revised form, March 5, 2010.

2010 Mathematics Subject Classification. Primary 35S05, 58J40.

(C)2010 American Mathematical Society Reverts to public domain 28 years from publication 
pseudodifferential operators defined microlocally in $U$ filtered by the order in $h$, and similarly for $V$ (see $₫ 2$ for definitions).

Theorem 1. Suppose

$$
g: \Psi^{0} / \Psi^{-\infty}(U) \rightarrow \Psi^{0} / \Psi^{-\infty}(V)
$$

is an order preserving algebra isomorphism. For every $\widetilde{U} \Subset U$ open, there is a symplectomorphism

$$
\kappa: \overline{\widetilde{U}} \rightarrow \overline{\kappa(\widetilde{U})}
$$

and $h_{0}>0$ such that, if $F$ is a microlocally unitary $h$-FIO associated to $\kappa$, then for all $0<h<h_{0}$ and all $P \in \Psi^{0} / \Psi^{-\infty}(U)$ we have

$$
g(P)=B F P F^{-1} B^{-1} \text { microlocally in } \kappa(\widetilde{U}) \times \widetilde{U},
$$

where $B \in \Psi^{0}(V)$ is elliptic.

Remark. A few remarks are needed about Theorem 1 First, this paper concerns operators which are defined microlocally in a precompact open subset of $T^{*} X$ (see $₫ 2$ for definitions). In keeping with the theme of working up to microlocal equivalence, Theorem 1 is really the analogue of Lemma 2 in DuSi 1 In proving Theorem 1 of DuSi], the authors take pains to remove the residual class $\Psi^{-\infty}$ from the statement of the theorem, which we do not do in this paper. In any applications of Theorem 1 in the present paper, we would first cut off to a precompact neighbourhood so Theorem 1 applies, then fix $B$ and $F$ (up to microlocal equivalence) and then deal with the errors from both the residual class and the cutoffs separately.

Second, there are two places in which the assumption $H^{1}(U, \mathbb{C})=0$ is used. One is that the $h$-FIO $F$ in Theorem 1 is constructed via deformation in the proof of Proposition 2.1. This deformation proof requires $H^{1}(U, \mathbb{C})=0$, although there are examples in which $H^{1}(U, \mathbb{C}) \neq 0$ and symplectomorphisms which can be quantized as an $h$-FIO (see Section 4 for an example). The second place where this assumption is used is in the construction of the pseudodifferential operator $B$, where we need to conclude that a closed 1-form is exact to construct the symbol of $B$.

Third, as the construction of the $h$-FIO is by deformation and only valid in a neighbourhood in phase space, we say $F$ is only microlocally defined. The operator $F$ is unique up to $\varnothing\left(h^{2}\right)$, and, once $F$ is fixed, $B$ is unique up to $\varnothing\left(h^{\infty}\right)$.

Fourth, to put Theorem 1 in context, we observe that every algebra homomorphism of the form (1.1) is an order preserving algebra isomorphism (see Proposition 2.1 below).

Finally, automorphisms of algebras of pseudodifferential operators have been studied in the context of the more abstract Berezin-Toeplitz quantization in [Zel, as well as for the Weyl algebra on Poisson manifolds in [B-KKo].

\section{Preliminaries}

Let $\mathcal{C}^{\infty}\left(T^{*} X\right)$ denote the algebra of smooth, $\mathbb{C}$-valued functions on $T^{*} X$, and define the global symbol classes

$$
\mathcal{S}^{m}\left(T^{*} X\right)=\left\{a \in \mathcal{C}^{\infty}\left(\left(0, h_{0}\right]_{h} ; \mathcal{C}^{\infty}\left(T^{*} X\right)\right):\left|\partial_{x, \xi}^{\alpha} a\right| \leq C_{\alpha} h^{-m}\right\} .
$$

\footnotetext{
${ }^{1}$ The statement of Theorem 10 is nevertheless simpler than [DuSi, Lemma 2] as in this case there are no transmissions. See the remark after Lemma 3.3 for an explanation of this.
} 
We define the essential support of a symbol by complement:

$$
\begin{aligned}
& \operatorname{ess-supp}_{h}(a) \\
& \quad=\complement\left\{(x, \xi) \in T^{*} X:\left|\partial_{x, \xi}^{\alpha} a\right| \leq C_{\alpha} h^{N} \forall N \text { and } \forall\left(x^{\prime}, \xi^{\prime}\right) \text { near }(x, \xi)\right\} .
\end{aligned}
$$

By multiplying elements of $S^{m}\left(T^{*} X\right)$ by an appropriate cutoff in $\mathcal{C}_{c}^{\infty}(U)$, we may think of symbols as being microlocally defined in $U$ and define the class of symbols with essential support in $U$ :

$$
\mathcal{S}^{m}(U)=\left\{a \in \mathcal{C}^{\infty}\left((0,1]_{h} ; \mathcal{C}_{c}^{\infty}(U)\right):\left|\partial_{x, \xi}^{\alpha} a\right| \leq C_{\alpha} h^{-m}\right\} .
$$

We write $\mathcal{S}^{m}=\mathcal{S}^{m}(U)$ when there is no ambiguity.

In keeping with the theme of defining everything up to microlocal equivalence, we recall Borel's lemma, which says for every sequence $a_{j} \in \mathcal{S}^{m_{j}}, j \geq 0$, with $m_{j}>m_{j+1}$ and $m_{j} \rightarrow-\infty$, there is an $a \in \mathcal{S}^{m_{0}}$ which is asymptotic to the sum

$$
a(x, \xi ; h) \sim \sum_{j=0}^{\infty} a_{j}(x, \xi ; h) .
$$

Here, "asymptotic to" means that for every $N \geq 0$,

$$
a(x, \xi ; h)-\sum_{j=0}^{N} a_{j}(x, \xi ; h) \in \mathcal{S}^{m_{N+1}} .
$$

By $\mathcal{S}^{0} / \mathcal{S}^{-1}$ we denote the set of equivalence classes of symbols, where $a$ is equivalent to $b$ if $a-b \in \mathcal{S}^{-1}$.

We have the corresponding spaces of pseudodifferential operators $\Psi^{m}(U)$ acting by the local formula (Weyl calculus)

$$
\mathrm{Op}_{h}^{w}(a) u(x)=\frac{1}{(2 \pi h)^{n}} \int_{\mathbb{R}^{n}} \int_{\mathbb{R}^{n}} a\left(\frac{x+y}{2}, \xi ; h\right) e^{i\langle x-y, \xi\rangle / h} u(y) d y d \xi .
$$

For $A=\mathrm{Op}_{h}^{w}(a)$ and $B=\mathrm{Op}_{h}^{w}(b), a \in \mathcal{S}^{m}, b \in \mathcal{S}^{m^{\prime}}$, we have the composition formula (see, for example, the review in $[\mathrm{DiSj}]$ )

$$
A \circ B=\mathrm{Op}_{h}^{w}(a \# b) \text {, }
$$

where

$$
\mathcal{S}^{m+m^{\prime}} \ni a \# b(x, \xi):=\left.e^{\frac{i h}{2} \omega\left(D_{x}, D_{\xi} ; D_{y}, D_{\eta}\right)}(a(x, \xi) b(y, \eta))\right|_{\substack{x=y \\ \xi=\eta}},
$$

with $\omega$ the standard symplectic form. Observe that \# preserves essential support

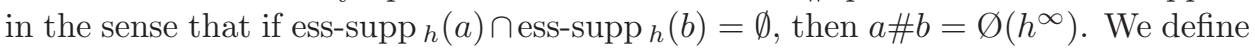
the wavefront set of a pseudodifferential operator $A=\mathrm{Op}_{h}^{w}(a)$ as

$$
\mathrm{WF}_{h}(A)=\operatorname{ess}^{-\operatorname{supp}_{h}(a)}
$$

so that $\Psi^{m}(U)$ is the class of pseudodifferential operators with wavefront set contained in $U$. We denote

$$
\Psi^{0}(U):=\bigcup_{m \leq 0} \Psi^{m}(U)
$$

and

$$
\Psi^{-\infty}(U):=\bigcap_{m \in \mathbb{Z}} \Psi^{m}(U)
$$


We will need the definition of microlocal equivalence of operators. Suppose $T: \mathcal{C}^{\infty}(X) \rightarrow \mathcal{C}^{\infty}(X)$ and that for any seminorm $\|\cdot\|_{1}$ on $\mathcal{C}^{\infty}(X)$ there is a second seminorm $\|\cdot\|_{2}$ on $\mathcal{C}^{\infty}(X)$ such that

$$
\|T u\|_{1}=\varnothing\left(h^{-M_{0}}\right)\|u\|_{2}
$$

for some $M_{0}$ fixed. Then we say $T$ is semiclassically tempered. We assume for the rest of this paper that all operators satisfy this condition (see [EvZw, Chap. 10] for more on this). Let $U, V \subset T^{*} X$ be open precompact sets. We think of operators defined microlocally near $V \times U$ as equivalence classes of tempered operators. The equivalence relation is

$$
T \sim T^{\prime} \Longleftrightarrow A\left(T-T^{\prime}\right) B=\varnothing\left(h^{\infty}\right): \mathcal{D}^{\prime}(X) \rightarrow \mathcal{C}^{\infty}(X)
$$

for any $A, B \in \Psi_{h}^{0,0}(X)$ such that

$$
\begin{aligned}
& \mathrm{WF}_{h}(A) \subset \widetilde{V}, \quad \mathrm{WF}_{h}(B) \subset \widetilde{U}, \text { with } \widetilde{V}, \widetilde{U} \text { open and } \\
& \bar{V} \Subset \widetilde{V} \Subset T^{*} X, \quad \bar{U} \Subset \widetilde{U} \Subset T^{*} X .
\end{aligned}
$$

In the course of this paper, when we say $P=Q$ microlocally near $U \times V$, we mean for any $A$ and $B$ as above,

$$
A P B-A Q B=\varnothing_{L^{2} \rightarrow L^{2}}\left(h^{\infty}\right),
$$

or in any other norm by the assumed precompactness of $U$ and $V$. Similarly, we say $B=T^{-1}$ on $V \times V$ if $B T=I$ microlocally near $U \times U$ and $T B=I$ microlocally near $V \times U$. Thus

$$
\Psi^{0} / \Psi^{-\infty}(U)
$$

is the algebra of bounded semiclassical pseudodifferential operators defined microlocally in $U$ modulo this equivalence relation. It is interesting to observe that this equivalence relation has a different meaning in the high-frequency regime. There, $\Psi^{-\infty}(X)$ corresponds to smoothing operators, although they may not be "small" in the sense of $h \rightarrow 0$.

We have the principal symbol map

$$
\sigma_{h}: \Psi^{m}(U) \rightarrow \mathcal{S}^{m} / \mathcal{S}^{m-1}(U),
$$

which gives the left inverse of $\mathrm{Op}_{h}^{w}$ in the sense that

$$
\sigma_{h} \circ \mathrm{Op}_{h}^{w}: \mathcal{S}^{m}(U) \rightarrow \mathcal{S}^{m} / \mathcal{S}^{m-1}(U)
$$

is the natural projection.

We will use the following well-known semiclassical version of Egorov's theorem (see Ch1, Ch2] or [EvZw for a proof).

Proposition 2.1. Suppose $U$ is an open neighbourhood of $(0,0)$ and $\kappa: \bar{U} \rightarrow \bar{U}$ is a symplectomorphism fixing $(0,0)$. Then there is a unitary operator $F: L^{2} \rightarrow L^{2}$ such that for all $A=\mathrm{Op}_{h}^{w}(a)$,

$$
A F=F B \text { microlocally on } U \times U,
$$

where $B=\mathrm{Op}_{h}^{w}(b)$ for a Weyl symbol b satisfying

$$
b=\kappa^{*} a+\varnothing\left(h^{2}\right) .
$$

$F$ is microlocally invertible in $U \times U$ and $F^{-1} A F=B$ microlocally in $U \times U$. 
Observe that Proposition 2.1 implicitly identifies a neighbourhood $U$ with its coordinate representation. In this paper, we will not use the fact that the error is $\varnothing\left(h^{2}\right)$, only that it is $\varnothing(h)$.

Let $Y$ be another smooth manifold of the same dimension as $X$, and let $V \subset T^{*} Y$ be a non-empty, precompact, open set. We say

$$
g: \Psi^{0} / \Psi^{-\infty}(U) \rightarrow \Psi^{0} / \Psi^{-\infty}(V)
$$

is an order preserving algebra isomorphism (of algebras filtered by powers of $h$ ) if

$$
g\left(\Psi^{m}(U)\right)=\Psi^{m}(V), g^{-1}\left(\Psi^{m}(V)\right)=\Psi^{m}(U),
$$

and for every $A, A^{\prime} \in \Psi^{m}(U), B \in \Psi^{m^{\prime}}(U)$,

$$
\begin{aligned}
g\left(A+A^{\prime}\right) & =g(A)+g\left(A^{\prime}\right) \bmod \Psi^{-\infty}(V), \\
g(A B) & =g(A) g(B) \bmod \Psi^{-\infty}(V) .
\end{aligned}
$$

\section{The Proof of Theorem 1}

We break the proof of Theorem 1 into several lemmas. The first lemma classifies maximal ideals of $\mathcal{S}^{0} / \mathcal{S}^{-1}+\mathbb{C}$ (which is the commutative algebra $\mathcal{S}^{0} / \mathcal{S}^{-1}$ with a unit added) in terms of certain equivalence classes of functions.

Lemma 3.1. The maximal ideals of $\mathcal{S}^{0} / \mathcal{S}^{-1}(U)+\mathbb{C}$ are either of the form

$$
\mathcal{M}_{\rho}:=\left\{p \in \mathcal{S}^{0} / \mathcal{S}^{-1}(U): p(\rho)=\varnothing(h), \rho \in U\right\}
$$

or

$$
\mathcal{M}_{\partial U}:=\mathcal{S}^{0} / \mathcal{S}^{-1}(U) .
$$

Proof. Clearly for each $\rho \in U, \mathcal{M}_{\rho}$ is a maximal ideal, as is $\mathcal{M}_{\partial U}$. In order to prove these are the only maximal ideals, suppose for contradiction that $\mathcal{M}$ is another maximal ideal which is not of the form (3.1) for any $\rho \in U$. We claim then that $\mathcal{M}_{\partial U} \subset \mathcal{M}$. To prove this, we observe that for each point $\rho \in U$, there is $a_{\rho} \in \mathcal{M}$ such that $\left|a_{\rho}(\rho)\right| \geq C^{-1}$ for some $C>0$ independent of $h$. Further, by multiplying by a constant if necessary, we may assume for each $\rho$ there is a neighbourhood $U_{\rho}$ of $\rho$ such that $\left.\operatorname{Re} a_{\rho}\right|_{\overline{U_{\rho}}} \geq 1$. Let $a(x, \xi) \in \mathcal{M}_{\partial U}$, and let

$$
K=\operatorname{ess-supp}_{h}(a) \Subset U \text {. }
$$

As $K$ is compact, we can cover it with finitely many of the $U_{\rho}$,

$$
K \subset U_{\rho_{1}} \cup \cdots \cup U_{\rho_{m}},
$$

and

$$
b:=\sum_{j=1}^{m} a_{\rho_{j}} \in \mathcal{M}
$$

satisfies $|b| \geq|\operatorname{Re} b| \geq 1$ on $K$. Thus $a / b \in \mathcal{M}_{\partial U}$ implies

$$
a=\left(\frac{a}{b}\right) b \in \mathcal{M} .
$$

Thus $\mathcal{M}_{\partial U} \subset \mathcal{M}$. But $\mathcal{M}_{\partial U}$ is maximal, so either $\mathcal{M}=\mathcal{M}_{\partial U}$ or $\mathcal{M}=\mathcal{S}^{0} / \mathcal{S}^{-1}(U)+$ $\mathbb{C}$.

The following three lemmas are a semiclassical version of DuSi with a few modifications to the proofs. 
Lemma 3.2. Suppose $g: \Psi^{0} / \Psi^{-\infty}(U) \rightarrow \Psi^{0} / \Psi^{-\infty}(V)$ is an order preserving algebra isomorphism. Then there exists a diffeomorphism $\kappa: U \rightarrow V$.

Proof. We first "unitalize" our algebra of pseudodifferential operators by adding constant multiples of identity. That is, let

$$
\tilde{\mathcal{S}}^{m}(U)=\left\{a \in \mathcal{C}^{\infty}\left((0,1]_{h} ; \mathcal{C}_{c}^{\infty}(U)+\mathbb{C}\right):\left|\partial^{\alpha} a\right| \leq C_{\alpha} h^{-m}\right\},
$$

and let $\widetilde{\Psi}^{m}(U)=\mathcal{O} p_{h}^{w} \tilde{\mathcal{S}}^{m}(U)$. We extend $g$ to an isomorphism

$$
\tilde{g}: \widetilde{\Psi}^{0} / \Psi^{-\infty}(U) \rightarrow \widetilde{\Psi}^{0} / \Psi^{-\infty}(V)
$$

by defining, for $C \in \mathbb{C}$ and $P \in \Psi^{0}(U)$,

$$
\tilde{g}(C+P):=C+g(P) .
$$

Observe that $\tilde{g}$ induces an algebra isomorphism

$$
g_{0}: \tilde{\mathcal{S}}^{0} / \mathcal{S}^{-1}(U)=\mathcal{S}^{0} / \mathcal{S}^{-1}(U)+\mathbb{C} \rightarrow \tilde{\mathcal{S}}^{0} / \mathcal{S}^{-1}(V)=\mathcal{S}^{0} / \mathcal{S}^{-1}(V)+\mathbb{C} .
$$

Since $g_{0}$ is an algebra isomorphism, it takes maximal ideals to maximal ideals. We observe that since $g_{0}: \mathcal{S}^{0} / \mathcal{S}^{-1}(U) \rightarrow \mathcal{S}^{0} / \mathcal{S}^{-1}(V)$,

$$
g_{0}\left(\mathcal{M}_{\partial U}\right)=\mathcal{M}_{\partial V}
$$

We can now define a map

$$
\kappa: U \rightarrow V
$$

by the following: for $\rho \in U$, define $\kappa(\rho)$ by

$$
g_{0}\left(\mathcal{M}_{\rho}\right)=\mathcal{M}_{\kappa(\rho)} .
$$

By applying $g_{0}^{-1}$, we immediately see $\kappa$ is bijective.

Now for $p \in \mathcal{S}^{0}(U)$ and $\rho \in U$, observe that

$$
p-p(\rho) \cdot 1 \in \mathcal{M}_{\rho}
$$

implies

$$
g_{0}(p)-p(\rho) \cdot 1 \in \mathcal{M}_{\kappa(\rho)} .
$$

Thus

$$
g_{0}(p)(\kappa(\rho))=p(\rho)+\varnothing(h)
$$

for every $\rho \in U$ implies

$$
g_{0}(p)=p \circ \kappa^{-1}+\varnothing_{p}(h),
$$

where $\varnothing_{p}(h)$ depends on $p$ but is smooth and uniformly bounded on $U$ since $p$ has compact support. Hence as a map $\mathcal{S}^{0} / \mathcal{S}^{-1}(U) \rightarrow \mathcal{S}^{0} / \mathcal{S}^{-1}(V)$,

$$
g_{0}(p)=p \circ \kappa^{-1} \text {. }
$$

For each $\rho \in U$, let $(x, \xi)$ be local coordinates for $U$ in a neighbourhood of $\rho$ which does not meet $\partial U$. Choosing a suitable cutoff $\chi_{\rho}$ equal to 1 near $\rho$, the $\chi_{\rho} x_{j}$ and $\chi_{\rho} \xi_{k}$ are approximate coordinates near $\rho$ :

$$
\begin{aligned}
& \chi_{\rho} x_{j}, \chi_{\rho} \xi_{k} \in \mathcal{S}^{0}(U) \text { for all } j, k \\
& \chi_{\rho} x_{j}=x_{j}, \chi_{\rho} \xi_{k}=\xi_{k} \text { near } \rho .
\end{aligned}
$$

Thus

$$
\left(\chi_{\rho} x_{j}\right) \circ \kappa^{-1} \in \mathcal{S}^{0}(V)
$$


and similarly for $\chi_{\rho} \xi_{j}$ for all $j$. Composing with inverse coordinate functions in a neighbourhood of $\kappa(\rho)$ implies $\kappa^{-1}$ is smooth on $U$. The same argument applied to $g^{-1}$ shows $\kappa$ is smooth on $V$, hence a diffeomorphism.

Lemma 3.3. The diffeomorphism $\kappa$ constructed in Lemma 3.2 is symplectic.

Remark. We observe that in this lemma, the phenomena of transmissions do not occur as in [DuSi, Lemma 2]. In that case, the diffeomorphism constructed using the point ideals as in Lemma 3.2 is a diffeomorphism $S^{*} X \rightarrow S^{*} Y$ where $X$ and $Y$ are $n$ dimensional manifolds, and the point ideals are contained in the algebra of functions which are homogeneous of degree 0 . It is in proving that the diffeomorphism lifts to $T^{*} X \backslash\{0\}$ by studying the action on functions homogeneous of degree 1 that an ambiguity is introduced. Since we are a priori working with open sets in $T^{*} X$ and smooth functions with no homogeneity assumption, no such ambiguity arises here. To see the relationship between the present result and that in [DuSi, Lemma 2], by considering symbols homogeneous of degree $1 \mathrm{in}$, say, a conic neighbourhood of a point in $S^{*} X$, and rescaling $h=|\xi|^{-1}$, we can apply the present result to get a localized version of DuSi, Lemma 2]. But the ambiguity of the sign of $\xi$ in the rescaling means that if we want to lift $\kappa$ to a diffeomorphism which commutes with multiplication in the fibers, $\kappa$ becomes either a homogeneous symplectomorphism or a homogeneous symplectomorphism composed with the map $(x, \xi) \mapsto(x,-\xi)$, as in DuSi.

Proof of Lemma 3.3. Observe that $\Psi^{0} / \Psi^{-\infty}(U)$ is a Lie algebra with brackets $i h^{-1}[\cdot, \cdot]$ and $g$ induces a Lie algebra isomorphism with $\Psi^{0} / \Psi^{-\infty}(V)$. Here $\mathcal{S}^{0}(U)$ is a Lie algebra with brackets $\{\cdot, \cdot\}$; hence $g_{0}$ is a Lie algebra isomorphism $\mathcal{S}^{0} / \mathcal{S}^{-1}(U)$ $\rightarrow \mathcal{S}^{0} / \mathcal{S}^{-1}(V)$. Let $a, b \in \mathcal{S}^{0}(U)$ and calculate

$$
g_{0}(\{a, b\})=\left\{g_{0}(a), g_{0}(b)\right\}
$$

or

$$
(\{a, b\}) \circ \kappa^{-1}=\left\{a \circ \kappa^{-1}, b \circ \kappa^{-1}\right\} .
$$

Letting $a$ and $b$ run through local approximate coordinates implies $\kappa^{-1}$ is symplectic.

Now fix $\widetilde{U} \Subset U$, and let

$$
F: L^{2}(\widetilde{U}) \rightarrow L^{2}(\widetilde{V})=L^{2}(\kappa(\widetilde{U}))
$$

be a microlocally unitary $h$-Fourier integral operator associated to $\kappa$ as in Proposition 2.1. We define an automorphism of $\Psi^{0} / \Psi^{-\infty}(\widetilde{U}), g_{1}$, by

$$
g_{1}(P)=F^{-1} g(P) F .
$$

Observe that $g_{1}$ is both order preserving and preserves principal symbol.

Lemma 3.4. Suppose

$$
g_{1}: \Psi^{0} / \Psi^{-\infty}(\widetilde{U}) \rightarrow \Psi^{0} / \Psi^{-\infty}(\widetilde{U})
$$

is an order-preserving automorphism which preserves principal symbol. Then there exists $B \in \Psi^{0}(\widetilde{U})$, elliptic on $\widetilde{U}$, such that

$$
g_{1}(P)=B P B^{-1} \bmod \varnothing\left(h^{\infty}\right)
$$

for every $P \in \Psi^{0} / \Psi^{-\infty}(U)$. 
Proof. The proof will be by induction, constructing $B$ in orders of $h$. We then use Borel's lemma to conclude that $B$ is asymptotic to the series in these orders of $h$, up to microlocal equivalence. We drop the dependence on $\widetilde{U}$ since the lemma is concerned with automorphisms. Suppose for $l \geq 1$ we have for every $m$ and every $P \in \Psi^{m}$

$$
g_{1}(P)-P \in \Psi^{m-l}
$$

This induces a map

$$
\beta: \mathcal{S}^{m} / \mathcal{S}^{m-1} \rightarrow \mathcal{S}^{m-l} / \mathcal{S}^{m-l-1},
$$

which, using the Weyl composition formula (2.1), satisfies

$$
\begin{aligned}
& \text { (i) } \beta(p q)=\beta(p) q+p \beta(q) \text {; } \\
& \text { (ii) } \beta(\{p, q\})=\{\beta(p), q\}+\{p, \beta(q)\} \text {. }
\end{aligned}
$$

Consider the action of $\beta$ on $\mathcal{S}^{0}$, and observe from property (i) above, for $p, q \in \mathcal{S}^{0}$,

$$
\beta(p q)=\beta(p) q+p \beta(q) \in \mathcal{S}^{-l},
$$

so $\beta$ is $h^{l}$ times a derivation on $\mathcal{S}^{0}$.

For any $\rho \in U$, we choose coordinates $(x, \xi)$ near $\rho$ and a cutoff $\chi_{\rho}$ which is equal to 1 near $\rho$ and compactly supported in $U$. Then $\chi_{\rho} x_{j}$ and $\chi_{\rho} \xi_{j}$ become approximate coordinates which are equal to $x_{j}$ and $\xi_{j}$ near $\rho$ but which are in $\mathcal{S}^{0}$. Near $\rho, \beta$ takes the form

$$
\beta=h^{l} \sum_{j}\left(\gamma_{j}(x, \xi) \partial_{x_{j}}+\delta_{j}(x, \xi) \partial_{\xi_{j}}\right)
$$

where $\gamma_{j}=h^{-l} \beta\left(\chi_{\rho} x_{j}\right)$ and $\delta_{j}=h^{-l} \beta\left(\chi_{\rho} \xi_{j}\right)$ (here $\gamma_{j}$ and $\delta_{j}$ implicitly depend on $h$, although we do not explicitly write it). Using property (ii) above, we have near $\rho$

$$
\beta\left(\left\{\chi_{\rho} x_{j}, \chi_{\rho} \xi_{k}\right\}\right)=\beta\left(\left\{\chi_{\rho} x_{j}, \chi_{\rho} x_{k}\right\}\right)=\beta\left(\left\{\chi_{\rho} \xi_{j}, \chi_{\rho} \xi_{k}\right\}\right)=0,
$$

which implies

$$
\frac{\partial \gamma_{j}}{\partial x_{k}}=-\frac{\partial \delta_{k}}{\partial \xi_{j}}, \frac{\partial \gamma_{j}}{\partial x_{k}}=\frac{\partial \gamma_{k}}{\partial x_{j}}, \text { and } \frac{\partial \delta_{j}}{\partial \xi_{k}}=\frac{\partial \delta_{k}}{\partial \xi_{j}} .
$$

Thus there exists a locally defined smooth function $f$ such that

and locally

$$
\gamma_{j}=\frac{\partial f}{\partial \xi_{j}} \text { and } \delta_{k}=-\frac{\partial f}{\partial x_{k}}
$$

$$
h^{-l} \beta=H_{f} .
$$

The function $f$ can be extended to be defined on all of $U$ by the assumption that $H^{1}(U, \mathbb{C})=0$. Define a smooth function $b$ by

$$
b=\exp (-i \chi f) \text {, }
$$

for a cutoff $\chi$ which is identically 1 on $\widetilde{U}$ with support in $U$, so that $d f=i d b / b$ on $\widetilde{U}$, and

$$
\beta=h^{l} H_{i \log b}
$$

Let $B=\mathrm{Op}_{h}^{w}(b)$ and observe that the principal symbol of

$$
B^{-1} P B-P=B^{-1}[P, B]
$$


in the $\mathcal{S}^{0}(\widetilde{U})$ calculus is

$$
\frac{h}{i} b^{-1}\{p, b\}=h H_{i \log b}(p) .
$$

For the base case of our induction, if $P \in \mathcal{S}^{m}(\widetilde{U})$, then

$$
g_{1}(P)-B^{-1} P B \in \mathcal{S}^{m-2}(\widetilde{U}),
$$

so that

$$
B g_{1}(P) B^{-1}-P \in \mathcal{S}^{m-2}(\widetilde{U}) .
$$

Replace $g_{1}(P)$ with $B g_{1} B^{-1}$.

Now for the purposes of induction, assume

$$
g_{1}(P)-P \in \mathcal{S}^{m-l}(\widetilde{U}),
$$

and apply the above argument to get $B_{l} \in \mathcal{S}^{-l}$ so that

$$
g_{1}(P)-B_{l}^{-1} P B_{l} \in \mathcal{S}^{m-l-1}(\widetilde{U}) .
$$

Then replacing $g_{1}(P)$ with $B_{l} g_{1}(P) B_{l}^{-1}$ finishes the induction. Thus there exists $B \in \mathcal{S}^{0}(\widetilde{U})$ so that

$$
B g_{1}(P) B^{-1}=P \bmod \varnothing\left(h^{\infty}\right) .
$$

Theorem 1 now follows immediately from applying Proposition 2.1 to (3.3) and (3.4).

\section{An EXAMPLE}

The current paper (and indeed the paper of Duistermaat and Singer DuSi on which this paper is based) largely skirts a very important related question by assuming $H^{1}(U, \mathbb{C})=0$. In this section we briefly consider a simple example which helps illustrate the necessity of this condition in general. We consider the following simple question: given a symplectomorphism $\kappa: U \rightarrow \kappa(U)$, is there a unitary operator which quantizes $\kappa$ ? More precisely, we investigate whether or not there is a unitary $h$-FIO which satisfies an Egorov type law.

Specifically, if $\kappa: U \rightarrow \kappa(U)$ is a symplectomorphism, we say a (microlocally) unitary $h$-FIO $F$ is of Egorov type if it satisfies a sharp Egorov conjugation law in the Weyl calculus: for $A \in \Psi_{h}^{l, 0}$,

$$
A(x, h D) F=F B(x, h D)
$$

where $\sigma(B)=\kappa^{*} \sigma(A)+e_{A}$, with

$$
e_{A} \in \mathcal{S}^{l-2,-2},
$$

with microsupport contained in the microsupport of $\kappa^{*} \sigma(A)$ (see Ch1 for notation and definitions). In the microlocal setting discussed in the previous sections, $U$ is precompact, so this just means $\mathcal{S}^{l, m}(U)=\mathcal{S}^{m}(U)$ in the notation from the previous sections, and hence $e_{A}=\varnothing\left(h^{2}\right)$.

\footnotetext{
${ }^{2}$ Here "unitary" refers to unitary on a fixed Hilbert space, typically $L^{2}$ of the base space, or of a compact subset thereof. "Microlocally" refers to the possibility that the Hilbert space is weighted with some pseudodifferential cutoffs.
} 
Proposition 4.1. Suppose $X=\mathbb{S}^{1}, U=T^{*} X \simeq S S_{x}^{1} \times \mathbb{R}_{\xi}$ equipped with the standard symplectic structure, and

$$
\kappa(x, \xi)=(x, \xi+\beta)
$$

for $\beta \in \mathbb{R}$. Then there exists an $h$-FIO $F$ of Egorov type quantizing $\kappa$, unitary on $L^{2}\left(S S^{1}\right)$, if and only if $\beta \in h \mathbb{Z}$.

Remark. Pullback by the symplectomorphism $\kappa$ from Proposition 4.1 clearly defines an order-preserving automorphism of the symbol algebra, preserving both the order in $h$ and in $\xi$. If we microlocalize everything in the following proof, that means it is order preserving in $h$ as discussed in the previous sections.

Proposition 4.1 should come as no surprise. In fact, from the point of view of geometric quantization, there is a pre-quantization for every element in $H^{1}\left(U, \mathbb{T}^{1}\right)$, the first cohomology group with coefficients in the unitary group. They differ by an overall phase factor of $e^{i 2 \pi \beta / h}$ for $0 \leq \beta<h$. That is, for $\beta \notin h \mathbb{Z}$, conjugation by a unitary $h$-FIO quantizing $\kappa$ is an isomorphism between inequivalent pre-quantizations and hence should not be an automorphism.

Proof. If $\beta \in h \mathbb{Z}$, then there is a global generating function for $\kappa$, and it is trivial to quantize $\kappa$. In fact, a unitary $h$-FIO quantizing $\kappa$ is given by multiplication by $e^{i \beta x / h}$, which is certainly $2 \pi$-periodic, unitary, and satisfies an exact Egorov type transformation law (see (4.2) below).

The proof of the reverse implication proceeds by contradiction. If there is such an operator, we lift it to the universal covering space and, by an approximate uniqueness result, we conclude that the operator must be given by multiplication by $e^{i \beta(x+2 \pi j) / h}$ for some $j \in \mathbb{Z}$, modulo a lower order term. But multiplication by $e^{i \beta(x+2 \pi j) / h}$ preserves periodicity of a function if and only if $\beta \in h \mathbb{Z}$.

For the purposes of contradiction, assume $\beta \notin h \mathbb{Z}$ and suppose there is nevertheless a unitary Egorov type $h$-FIO $F$ quantizing $\kappa$. That is, for $u(x) \in L^{2}\left(S S^{1}\right)$, $F u \in L^{2}\left(S S^{1}\right)$ with $\|F u\|=\|u\|, F^{*}=F^{-1}$, and for all pseudodifferential operators $A \in \Psi_{h}^{l, 0}\left(S S^{1}\right)$, there is a pseudodifferential operator $e_{A} \in \Psi_{h}^{l-2,-2}\left(S S^{1}\right)$ so that

$$
A F=F\left(\tilde{A}+e_{A}\right),
$$

where $\sigma(\tilde{A})(x, \xi)=\kappa^{*} \sigma(A)(x, \xi)=\sigma(A)(x, \xi+\beta)$. The universal covering space of $S S^{1}$ is $\mathbb{R}$, so we want to compare $F$ to operators acting (locally) on $L^{2}(\mathbb{R})$. We regard $L^{2}\left(S S^{1}\right)$ as a vector subspace of $\mathcal{S}^{\prime}(\mathbb{R})$, that is, $2 \pi$-periodic functions on the real line which are square-integrable over one period. We lift $F$ to an operator on $L^{2}\left(S S^{1}\right) \subset \mathcal{S}^{\prime}(\mathbb{R})$ which is unitary over any interval of length $2 \pi$ by partitioning $\mathbb{R}$ into intervals of length bounded by $2 \pi$. That is, the lift of $F$ acting on a function supported in an interval of length $2 \pi$ is $F$ acting on the $2 \pi$-periodic extension. This is well-defined up to smoothing operators of order $h^{\infty}$ according to the assumption that $F$ is of Egorov type. We abuse notation and continue to call this lift $F$.

Fix $s_{0} \in \mathbb{R}$, and let $a(x) \in \mathcal{C}_{c}^{\infty}\left(\left[s_{0}, s_{0}+2 \pi\right]\right)$. Since $a$ is supported in one period, we have an Egorov transformation rule:

$$
a(x) F=F\left(a(x)+e_{a}\right)
$$

for $e_{a} \in \Psi_{h}^{-2,-2}\left(S S^{1}\right)$, since $\kappa$ is the identity in the $x$ variable. The operator $a(x) F$ now maps $L^{2}\left(S S^{1}\right)$ to $L^{2}\left(\left[s_{0}, s_{0}+2 \pi\right]\right)$ (with no periodic extension), and by (4.1), $a(x) F$ also maps $L^{2}\left(\left[s_{0}, s_{0}+2 \pi\right]\right)$ into $L^{2}\left(\left[s_{0}, s_{0}+2 \pi\right]\right)$. 
Consider now the multiplication operators

$$
F_{j}=e^{i \beta(x+2 \pi j) / h}
$$

for $j \in \mathbb{Z}$, acting on $L^{2}(\mathbb{R})$. Clearly $F_{j}$ is unitary on $L^{2}(I)$ for any interval $I \subset \mathbb{R}$, and $F_{j}$ satisfies the exact Egorov law

$$
\begin{aligned}
A(x, h D) F_{j} u(x) & =(2 \pi h)^{-1} \int e^{i(x-y) \xi / h} A((x+y) / 2, \xi) e^{i \beta(y+2 \pi j) / h} u(y) d y d \xi \\
& =(2 \pi h)^{-1} e^{i \beta(x+2 \pi j) / h} \int e^{i(x-y)(\xi-\beta) / h} A((x+y) / 2, \xi) u(y) d y d \xi \\
& =F_{j} A(x, h D+\beta) u(x) .
\end{aligned}
$$

Multiplying by the compactly supported function $a(x)$, we have

$$
a F_{j}=F_{j} a
$$

with no error term. If we choose $a$ real,

$$
\left(a F_{j}\right)^{*}=F_{j}^{*} a=e^{-i \beta(x+2 \pi j) / h} a=a e^{-i \beta(x+2 \pi j) / h},
$$

so that

$$
\begin{aligned}
(a F)\left(a F_{j}\right)^{*} & =a F a F_{j}^{*} \\
& =a^{2} F F_{j}^{*}-a F e_{a} F_{j}^{*} \\
& =\left(a^{2}-a \tilde{e}_{a}\right) F F_{j}^{*}
\end{aligned}
$$

for a pseudodifferential operator $\tilde{e}_{a} \in \Psi_{h}^{-2,-2}\left(\left[s_{0}, s_{0}+2 \pi\right]\right)$. We now claim that there is a constant $c_{j},\left|c_{j}\right|=1$, and a pseudodifferential operator $E_{a, j} \in \Psi_{h}^{-2,-2}(\mathbb{R})$ so that

$$
a^{2} F F_{j}^{*}=c_{j} a^{2}\left(I+E_{a, j}\right)
$$

as operators on $L^{2}\left(\left[s_{0}, s_{0}+2 \pi\right]\right)$. Indeed, we have that $a^{2} F F_{j}^{*} \in \Psi_{h}^{0,0}(\mathbb{R})$ is a semiclassical pseudodifferential operator,

$$
a^{2} F F_{j}^{*} x=a^{2}\left(x+e_{1}\right) F F_{j}^{*}, e_{1} \in \Psi_{h}^{-2,-2}(\mathbb{R}),
$$

and

$$
a^{2} F F_{j}^{*} h D=a^{2}\left(h D+e_{2}\right) F F_{j}^{*}, e_{2} \in \Psi_{h}^{-1,-2}(\mathbb{R}),
$$

which implies the principal and sub-principal symbol of $a^{2} F F_{j}^{*}$ is a constant times $a^{2}$. Since $\left(F F_{j}^{*}\right)^{*}=\left(F F_{j}^{*}\right)^{-1}$ on $\operatorname{supp}\left(a^{2}\right)$, we know this constant has modulus 1 .

This tells us that, at least for part of a period, $F$ is multiplication by $e^{i \beta(x+2 \pi j) / h}$ for some $j$, up to errors of size $h^{2}$. The next step is to show that this is true for a whole period. That is, let $a_{1}+a_{2} \equiv 1$ on $[0,2 \pi]$, but with each $a_{l}$ supported in an interval of length less than $2 \pi$ so that the above discussion applies to each $a_{l}$. Then we calculate

$$
\begin{aligned}
\left(a_{1}+a_{2}\right) F\left(c_{1} a_{1} F_{j_{1}}\right. & \left.+c_{2} a_{2} F_{j_{2}}\right)^{*} \\
& =\left(a_{1}+a_{2}\right) F\left(\overline{c_{1}} a_{1} F_{j_{1}}^{*}+\overline{c_{2}} a_{2} F_{j_{2}}^{*}\right) \\
& =\left(a_{1}+a_{2}\right) F\left(\overline{c_{1}} a_{1} e^{-i 2 \pi \beta j_{1} / h} F_{0}^{*}+\overline{c_{2}} a_{2} e^{-i 2 \pi \beta j_{2} / h} F_{0}^{*}\right) \\
& =\left(a_{1}+a_{2}\right)\left(\overline{c_{1}} a_{1} e^{-i 2 \pi \beta j_{1} / h}+\overline{c_{2}} a_{2} e^{-i 2 \pi \beta j_{2} / h}\right) F F_{0}^{*}
\end{aligned}
$$


plus errors of size $h^{2}$ which we neglect for the rest of the calculation. This operator now a priori is defined on $L^{2}(\mathbb{R})$, but if we compose with another function $b$ supported in $[0,2 \pi]$, but $b \equiv 1$ in a neighbourhood of supp $\left(a_{1}\right) \cap \operatorname{supp}\left(a_{2}\right)$, we conclude we must have

$$
\left(\overline{c_{1}} a_{1} e^{-i 2 \pi \beta j_{1} / h}+\overline{c_{2}} a_{2} e^{-i 2 \pi \beta j_{2} / h}\right)=1+\varnothing\left(h^{2}\right)
$$

on $\left\{a_{1}+a_{2}=1\right\}$. That is, replacing $a_{2}$ with $1-a_{1}$ on $\operatorname{supp}\left(a_{1}\right) \cap \operatorname{supp}\left(a_{2}\right)$, (4.4) must be independent of $a_{1}$ up to $\varnothing\left(h^{2}\right)$, so that

$$
\frac{c_{1}}{c_{2}}=e^{i 2 \pi \beta\left(j_{2}-j_{1}\right) / h}\left(1+\varnothing\left(h^{2}\right)\right),
$$

or $c_{1}$ and $c_{2}$ differ only by a phase shift, up to leading order. Without loss of generality, we write $c_{1}=1+\varnothing\left(h^{2}\right)$ and $c_{2}=e^{-i 2 \pi \beta j / h}\left(1+\varnothing\left(h^{2}\right)\right)$ in the calculation leading to (4.3) to get

$$
\left(a_{1}+a_{2}\right) F\left(a_{1} F_{0}+c_{2} a_{2} F_{0}\right)^{*}=\left(a_{1}+a_{2}\right)\left(a_{1}+e^{i 2 \pi \beta j / h} a_{2}\right) F F_{0}^{*} .
$$

But by the above argument this implies that either $j=0$ or $\beta=\varnothing\left(h^{3}\right)$.

This implies the $h$-FIO $F$ is equal to a unimodular constant times one of the $F_{j}$ on any interval of length $2 \pi$, which is a whole period, and hence $F$ cannot be periodic.

\section{ACKNOWLEDGEMENTS}

The author would like to thank Maciej Zworski for suggesting this problem and for many helpful conversations. This work was started while the author was a graduate student in the mathematics department at UC Berkeley, and he is very grateful for the support received while there. The author would also like to thank the anonymous referee, whose many insightful comments and corrections have greatly helped improve this paper, and for suggesting the clarifying example in Section 4

\section{REFERENCES}

[B-KKo] Belov-Kanel, A. And Kontsevich, M. Automorphisms of the Weyl Algebra. Lett. Math. Phys. 74 (2005), no. 2, 181-199. MR2191954(2006k:16068)

[Ch1] Christianson, H. Semiclassical Non-concentration Estimates Near Hyperbolic Orbits. J. Funct. Anal. 262 (2007), no. 2, 145-195. MR2321040(2008k:58058)

[Ch2] Christianson, H. Quantum Monodromy and Non-concentration Near a Closed Semihyperbolic Orbit. To appear, Trans. Amer. Math. Soc.

[DiSj] Dimassi, M. And SJöstrand, J. Spectral Asymptotics in the Semi-classical Limit. Cambridge University Press, Cambridge, 1999. MR.1735654 (2001b:35237)

[DuSi] Duistermat, J. J. And Singer, I. M. Order-Preserving Isomorphisms between Algebras of Pseudo-Differential Operators. Commun. Pure Appl. Math. 29 (1976), 39-47. MR0402830 (53:6644)

[EvZw] Evans, L.C. AND Zworski, M. Lectures on Semiclassical Analysis. http://math. berkeley.edu/ evans/semiclassical.pdf.

[Zel] Zelditch, S. Quantum Maps and Automorphisms. The breadth of symplectic and Poisson geometry, Prog. Math., 232, Birkhäuser Boston (2005), 623-654. MR2103022 (2005j:53113)

Department of Mathematics, Massachusetts Institute of Technology, 77 MassachuSetts Avenue, Cambridge, Massachusetts 02139-4307

Current address: Department of Mathematics, University of North Carolina, Chapel Hill, North Carolina 27599-3250

E-mail address: hans@math.mit.edu 